\title{
Sentidos de vida de los universitarios ${ }^{1}$
}

\section{María Victoria Cadavid-Claussen}

Universidad Católica de Colombia (Colombia)

mvcadavid@ucatolica.edu.co

\section{Víctor Manuel Díaz-Soto}

Universidad Católica de Colombia (Colombia)

vmdiaz@ucatolica.edu.co

\section{Resumen}

Esta investigación contribuye a la identificación de los factores que motivan la configuración del sentido de vida en su dinámica de adquisición, y propone una aproximación metodológica para una mejor comprensión y educación del sentido de vida personal en los jóvenes universitarios. La investigación formula dos categorías: los radicales antropológicos del sentido, que provienen de la condición interior o intimidad personal, y las líneas de orientación al sentido, que son existenciales y se realizan como distintos ámbitos vitales. A partir del cruce de estas dos dimensiones, según el mayor o menor grado de apertura de los radicales personales y de despliegue vital de los ámbitos existenciales, surgen nueve tipos de sentido de vida. Con este modelo conceptual se presentan dos instrumentos, un cuestionario y una entrevista focalizada, que se han aplicado en una muestra de 720 jóvenes universitarios de ocho universidades de Bogotá. El hallazgo principal de este estudio muestra que los sentidos de vida de los jóvenes universitarios son subjetivos, más construidos de cara al horizonte futuro que extraídos o fundados en la experiencia vital, pero en todo caso, cargados de los valores que asume, en relevo, una generación.

\section{Palabras clave}

Estudiante universitario, investigación social, antropología de la educación, tipología (Fuente: Tesauro de la Unesco).

$1 \quad$ Esta investigación se desarrolló como tesis de grado de la Maestría en Educación de la Universidad de La Sabana en el año 2009, con el apoyo de la Universidad Católica de Colombia.

Recepción: 19-06-2014 | Envío a pares: 22-08-2014 | Aceptación por pares:20-07-2015 | Aprobación:15-08-2015 


\title{
The Meaning of Life for University Students
}

\begin{abstract}
This research helps to identify the factors that motivate the dynamics involved in how meaning of life is acquired and proposes a methodological approach to improve understanding and education on the personal meaning of life for university students. The study lays out two categories: the anthropological radicals of that meaning, which come from within or personal intimacies, and the lines of orientation towards the meaning of life, which are existential and realized as different spheres in one's experience. Nine types of meaning in life emerge from the intersection of these two dimensions, depending on the greater or lesser degree of openness in personal radicals and how life plays out in its existential fields. Two tools were used with this conceptual model: a questionnaire and a focused interview. They were applied to a sample of 720 university students from eight universities in the city of Bogotá (Colombia). Essentially, this study shows the meaning of life for university students is subjective and constructed more with an eye towards the future than based on or extracted from their experiences in life, but is laden invariably with the values assumed in a generational shift.
\end{abstract}

\section{Keywords}

College student, social research, anthropology of education, type (Source: Unesco Thesaurus). 


\section{Sentidos de vida dos universitários}

\section{Resumo}

Esta pesquisa contribui para a identificação dos fatores que motivam a configuração do sentido de vida em sua dinâmica de aquisição, e propõe uma aproximação metodológica para uma melhor compreensão e educação do sentido de vida pessoal nos jovens universitários. A pesquisa formula duas categorias: os radicais antropológicos do sentido, que provêm da condição interior ou intimidade pessoal, e as linhas de orientação ao sentido, que são existenciais e se realizam como diferentes âmbitos vitais. A partir do cruzamento dessas duas dimensões, de acordo com o grau variável de abertura dos radicais pessoais e de desdobramento vital dos âmbitos existenciais, surgem nove tipos de sentido de vida. Com esse modelo conceitual apresentam-se dois instrumentos: um questionário e uma entrevista focalizada, que foram aplicados numa amostra de 720 jovens universitários de oito universidades de Bogotá. A principal conclusão deste estudo mostra que os sentidos de vida dos jovens universitários são subjetivos, que são mais construídos no horizonte futuro do que extraídos ou fundados na experiência vital, mas, em todo caso, carregados dos valores que assume, em relevo, uma geração.

\section{Palavras-chave}

Estudante universitário, pesquisa social, antropologia da educação, tipologia (Fonte: Tesauro da Unesco) (Fonte: Tesauro da Unesco). 


\section{Introducción}

La presente investigación enuncia un modelo de sentido originado por el cruce de dos ejes: en sentido vertical, los radicales antropológicos del sentido (RAS) que pueden ser inhibidos o desplegados; y el eje horizontal representa las líneas de orientación al sentido (LOS) que pueden ser orientadas al sentido o al contrasentido (LOCS). El cruce de estos ejes configura cuatro cuadrantes del sentido: objetivo, subjetivo antropológico, subjetivo existencial y contrasentido. Estos ejes, como ortogonales, pueden considerarse consistentes con las dimensiones del sentido de vida que se reconocen en las últimas investigaciones (Martínez, García-Alandete, Sellés, Bernabé y Soucase, 2012) (ver cuadro 1): coherencia existencial y propósito vital (Martínez, Trujillo, Díaz y Jaimes, 2011); satisfacción y sentido de la vida y, metas y propósitos vitales (García-Alandete, Rosa y Sellés, 2013).
Esta investigación está encaminada a determinar las tipologías de sentido de vida en los universitarios y establecer los factores que configuran dicho sentido en la población universitaria de Bogotá, según un modelo antropológico-existencial, desarrollado en un artículo previo que profundiza los contenidos antropológicos (Cadavid y Díaz, 2014). La investigación es cualitativa y fenomenológica (Castro, Castro y Morales, 2005), pues trata de indagar con los estudiantes universitarios de pregrado, desde sus vivencias, los factores antropológicos que inciden en la realización de su sentido de vida, sus líneas de orientación existencial y los tipos de sentido vital que manifiestan.

En esta línea apuntan las investigaciones humanistas-existenciales y sus conceptos claves en relación con las dimensiones del bienestar humano: madurez, ciclo y tendencias de vida, salud mental,

\section{Cuadro 1. Factores del sentido de vida de contenido relevante y significatividad en instrumentos (cuestionario y entrevista)}

\begin{tabular}{|c|c|c|c|c|c|}
\hline Autores & Factor 1 & Factor 2 & Factor 3 & Factor 4 & Factor 5 \\
\hline $\begin{array}{c}\text { McGregor y Little } \\
(1998)^{*}\end{array}$ & Felicidad & Sentido & & & \\
\hline $\begin{array}{l}\text { García-Alandete } \\
\text { et al. }(2011)^{*}\end{array}$ & $\begin{array}{l}\text { Satisfacción y } \\
\text { Sentido de vida }\end{array}$ & $\begin{array}{c}\text { Metas y } \\
\text { propósitos en la } \\
\text { vida }\end{array}$ & & & \\
\hline $\begin{array}{c}\text { Martínez, Trujillo, } \\
\text { Díaz y Jaimes } \\
(2011)^{* *}\end{array}$ & $\begin{array}{l}\text { Percepción de } \\
\text { propósito vital }\end{array}$ & $\begin{array}{l}\text { Coherencia } \\
\text { existencial }\end{array}$ & & & \\
\hline $\begin{array}{c}\text { Magaña et al. } \\
\qquad(2004)^{*}\end{array}$ & $\begin{array}{c}\text { Percepción } \\
\text { de sentido y } \\
\text { significado de la } \\
\text { vida }\end{array}$ & $\begin{array}{c}\text { Satisfacción por } \\
\text { la propia vida }\end{array}$ & $\begin{array}{l}\text { Libertad y control } \\
\text { de la propia vida }\end{array}$ & & \\
\hline $\operatorname{Risco}(2009)^{*}$ & Valor de la vida & $\begin{array}{l}\text { Capacidad de } \\
\text { significado }\end{array}$ & $\begin{array}{c}\text { Metasy } \\
\text { responsabilidad }\end{array}$ & & \\
\hline $\begin{array}{l}\text { Noblejas de la } \\
\text { Flor }(1994)^{*}\end{array}$ & $\begin{array}{l}\text { Percepción de } \\
\text { sentido }\end{array}$ & $\begin{array}{l}\text { Experiencia de } \\
\text { sentido }\end{array}$ & Metas y tareas & $\begin{array}{c}\text { Dialéctica } \\
\text { Destino/Libertad }\end{array}$ & \\
\hline $\begin{array}{c}\text { Cadavid y Díaz } \\
(2014)\end{array}$ & $\begin{array}{c}\text { Radicales } \\
\text { antropológicos }\end{array}$ & $\begin{array}{l}\text { Líneas de } \\
\text { orientación } \\
\text { existencial }\end{array}$ & $\begin{array}{c}\text { Actitud ante } \\
\text { situaciones límite }\end{array}$ & $\begin{array}{c}\text { Actitud ante el } \\
\text { cambio }\end{array}$ & $\begin{array}{l}\text { Actitud ante el } \\
\text { futuro }\end{array}$ \\
\hline
\end{tabular}


individuación, autoactualización y plenitud (de Lara y Ruschel, 2012).

\section{Estructura metodológica del modelo antropológico-existencial de sentido de vida}

En el plano antropológico-existencial del sentido (figura 1) se muestra, por una parte, la distancia polar entre los sentidos de vida objetivos y los contrasentidos, y por otra, que según el grado de despliegue de los criterios definidos se presentan nueve tipos de sentido.

Es una realidad existencial que cuando la persona realiza un despliegue positivo de su ser personal - eje vertical superior-, y al hallarse situada en una red de ámbitos que le facilitan orientarse al descubrimiento de sentido - eje horizontal derecho-, se encuentra más afianzada para enfrentar su situación existencial (Polo, 2005), para realizarla con una actitud responsable y realista según los requerimientos de su propio ser y de su entorno vital (Frankl, 2003).

Las tipologías de sentido de vida están vertidas en un plano cartesiano, cuyos ejes corresponden a LOS y RAS con rangos de variables definidos según las tablas de asignación de tipos de sentido correspondientes a las preguntas 1 y 2 del cuestionario.

En la figura 1 se observa cómo los tipos de sentido de vida emergen en el mapa existencial de la persona humana. Dada la infinitud de opciones de su despliegue libre y también por la dinámica permanente del existir (Abbagnano, 1997, p. 29), el sentido de vida aparece para la persona como un modo de ver su existencia y de resolverla; y así orienta su ruta vital y se autodetermina (Wojtyla, 2005, pp. 171185). El plano de los tipos de sentido permite el conocimiento de una especie de geografía del sentido arraigada en la profundidad significativa de sus dos

Figura 1. Expresión metodológica de las tipologías de sentido de vida en el plano antropológico-existencial

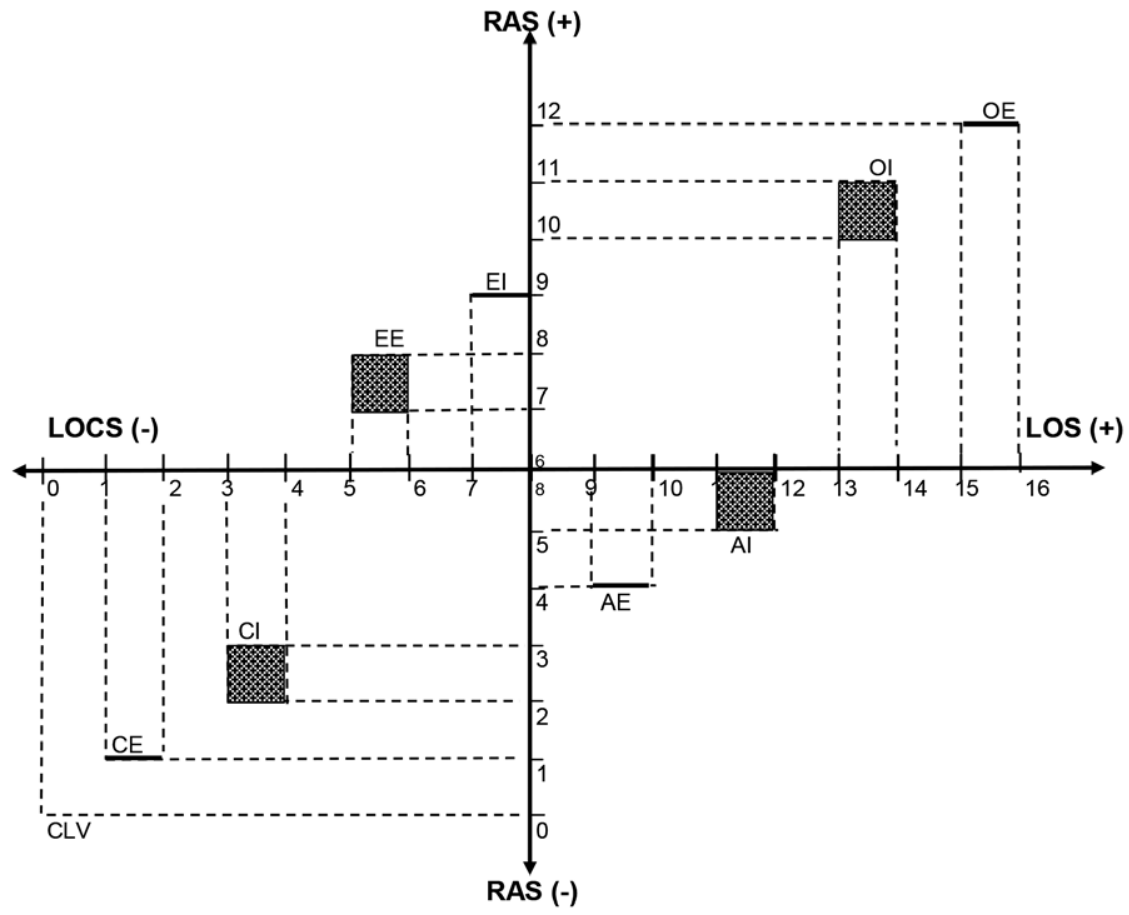


ejes, que constituyen el despliegue de la interioridad personal desde el núcleo personal, como centro del sentido, a través de la esencia, inteligencia y voluntad, por las que se es capaz de tejer la propia biografía, y contando con la materialidad de la naturaleza humana, como biología (Sellés, 2007). Esta dinámica de integridad de distinto nivel, que es bio-psico-espiritual (Frankl, 2001, p. 53) permite el despliegue y crecimiento personal desde los radicales antropológicos en los distintos ámbitos de la realidad existencial.

Aunque por el dinamismo humano esencial y existencial (Heidegger, 1999, p. 403) es posible migrar en el sentido, no es común, salvo alguna situación existencial extrema, el salto abrupto vital desde los tipos de sentido objetivos al contrasentido o viceversa. En cambio, los tipos de sentido subjetivos se observan más cercanos entre sí, aunque claramente diferenciados; aparecen más cerca de los ejes, y por ello con mayor posibilidad de intercambio de valores especialmente desde los tipos de sentido inestable. Esto es posible existencialmente cuando los valores de las dos dimensiones, antropológica y existencial, están más débiles. Sin embargo, es importante aclarar que el plano muestra los tipos de sentido puros, y que también sería posible observar, con una combinación más compleja de valores en las variables, cómo los tipos de sentido se amplían y colonizan un territorio más extenso de la geografía del sentido. Este dinamismo o movilidad de sentidos, suele acompañar a la persona durante su existencia. De aquí la importancia de defender o recuperar la claridad del centro personal que orienta la migración consistente hacia el sentido objetivo (Freire, 2007, pp. 294-300).

\section{Técnicas}

Se utilizaron dos técnicas. La entrevista en grupos focales (Martínez Miguelez, 2006, pp. 93-99), de 8 a 10 estudiantes, se realizó en siete universidades de Bogotá, con un total de 11 grupos focales y 87 universitarios entrevistados: dos en cada una de las universidades -Pontificia Universidad Javeriana,
Católica de Colombia, Minuto de Dios y Distrital-, y un grupo focal en las universidades Pedagógica, La Salle y La Sabana. La selección que se solicitó a cada universidad fue aleatoria en edad, sexo y programas académicos. La actitud de las universidades en general fue muy positiva y de gran apertura e interés, y sobre todo de enriquecimiento mutuo por el intercambio de experiencias, inquietudes y acciones.

También se utilizó la técnica del cuestionario individual (Ander-Egg, 2001), en veinte grupos de estudiantes de ocho universidades de Bogotá, con un total de 634 estudiantes de pregrado distribuidos así: seis grupos en la Pontificia Universidad Javeriana, tres grupos en la Universidad de La Sabana, tres en la Distrital, uno en la Pedagógica, cinco en La Salle, tres en Uniminuto, cinco en la Santo Tomás y cuatro en la Católica. Se decidió con las universidades que la estrategia pertinente consistiría en solicitar a algunos docentes su colaboración con sus grupos de estudiantes y que el instrumento se aplicaría en sus horas de clase. Se destaca el interés de los docentes por el tema de investigación y por conocer los resultados.

\section{Población}

Los 720 jóvenes participantes se encuentran en el centro de su vida universitaria. Están en la mitad de sus carreras, de modo que ya se encuentran bien instalados en el ambiente universitario y así se constituyen para el investigador en referentes claros de la tipología de sentido juvenil. La muestra comprende jóvenes de $3^{\circ}$ a $8^{\circ}$ semestre de las universidades mencionadas y que acceden voluntariamente a participar. Las edades oscilan entre los 18 y 24 años.

\section{Criterios de selección}

Los criterios de selección fueron accesibilidad, representatividad y oportunidad. Se escogieron las universidades que respondieron a la invitación hecha por el director de la Maestría de Educación de la Universidad de La Sabana a varias universidades de 
Bogotá, oficiales y privadas, y que logran reunir un grupo aleatorio de jóvenes participantes dentro del tiempo para ello determinado (noviembre de 2007 mayo de 2008).

\section{Instrumentos}

La entrevista focal responde a la necesidad inicial de sondear y acercarse a los jóvenes universitarios desde su experiencia del sentido de vida, propiciando que durante la conversación pueda surgir simultáneamente la autorreflexión (Wojtyla, 2005, p. 181) y el darse cuenta de los aspectos personales del sentido de vida y su realización en la existencia de cada uno (Seligman, 2003).

"Dado que la problemática del sentido en una vida término medio normal más bien está latente en la conciencia y solo toma cuerpo debido al estrés, a la frustración o al defecto extremo de tensión, durante la encuesta fue necesario atraerla directamente al plano consciente" (Lukas, 1994, p. 256).

Por otro lado, este instrumento permite a los investigadores recoger la posición de los jóvenes frente al sentido de vida como colectivo social (Spranguer, 1968). En el grupo es posible acceder al conocimiento de las posiciones sobre los ámbitos y factores del sentido de vida como se presenta en la dinámica de la realidad existencial del joven universitario y se proyecta en el plano de realización de sentido-fracaso (Frankl, 1984, p. 44).

Por otra parte, el cuestionario constituye el acercamiento personal al sentido existencial de vida, desde la propia experiencia biográfica. Aquí los investigadores reconocen la confidencialidad e intimidad que requiere el ejercicio de autoexamen y balance vital para descubrirse cada uno a sí mismo en su tipo de sentido de vida, desvelar el despliegue de sus radicales de sentido (García Pintos et al., 2007, pp.43-50) y su ubicación en los diferentes ámbitos de realización del mismo (Lukas, 2003). De este modo, se amplía a otros criterios el plano de realización de sentido-fracaso de Frankl (1984, p. 44).
Los instrumentos son validados por cuatro pares expertos, dos por cada instrumento, y sus observaciones coinciden en la necesidad de disminuir el número de preguntas agrupándolas según sus contenidos en referencia a cada uno de los criterios generales definidos para el diseño de los instrumentos. También se sugiere simplificar las preguntas organizándolas en cuadros de modo que incluyan las opciones de respuesta; finalmente, se recomienda que estas opciones de respuesta se aproximen en lo posible a la escala de Likert. Adicionalmente, se realiza una prueba piloto con un grupo de 11 estudiantes, hombres y mujeres entre 18 y 24 años, de distintos programas académicos y diferentes semestres.

En la estructura de prueba de la entrevista (cuadro 2) se puede observar la injerencia o no de los criterios generales propuestos en las distintas preguntas con la "X" mayúscula. Estos criterios son los siguientes: DO es el primer criterio que significa el despliegue de radicales antropológicos de sentido (RAS) y las líneas de orientación al sentido (LOS) o al contrasentido (LOCS); la actitud ante el valor (AV) es el modo objetivo o subjetivo como el joven se sitúa ante la presencia del sentido vital; la estabilidad vital (EV) representa la persistencia existencial del joven en varias líneas de orientación al sentido o al contrasentido; por último, el criterio del grado de interés vital (GIV) se refiere a la proporción de radicales antropológicos y de líneas de orientación al sentido existente en cada joven (Cadavid y Díaz, 2014).

Las palabras en cursiva en las preguntas de la estructura de prueba significan el énfasis que el entrevistador hace en la entrevista para conseguir las respuestas pertinentes.

Para cada entrevista focal se diligenció un "Formato de registro y análisis de entrevista grupal", con las respuestas de los participantes. En cada entrevista tomaron parte, además del entrevistador (uno de los dos investigadores principales), dos observadores auxiliares, de modo que cada uno de ellos se hizo cargo de registrar las respuestas de la mitad de los 


\section{Cuadro 2. Estructura de prueba de la entrevista focal "Tipos de sentido de vida en los jóvenes universitarios" (Cadavid-Díaz, 2014)}

\begin{tabular}{|c|c|c|c|c|}
\hline \multirow{2}{*}{ Guía de preguntas de la entrevista } & Crt1 & Crt2 & Crt3 & Crts \\
\hline & DO & AV & EV & GIV \\
\hline $\begin{array}{l}\text { 1. Cuando piensan en "Sentido de vida" qué es lo primero que se } \\
\text { les ocurre, qué palabras o expresiones les llegan a la mente. }\end{array}$ & $x$ & $x$ & & \\
\hline 2. ¿Qué hace que la vida del joven universitario tenga sentido? & $x$ & $x$ & $X$ & $X$ \\
\hline 3. ¿Qué hace que la vida del joven universitario pierda sentido? & $x$ & $x$ & $x$ & $x$ \\
\hline 4. ¿Qué requisitos creen necesarios para tener una vida realizada? & $x$ & $x$ & $x$ & $x$ \\
\hline 5. ¿Cuándo creen que una vida fracasa? & $x$ & $x$ & $x$ & $X$ \\
\hline $\begin{array}{l}\text { 6. ¿Hasta qué punto los jóvenes pueden definir el rumbo de sus } \\
\text { vidas? }\end{array}$ & & $x$ & & $x$ \\
\hline $\begin{array}{l}\text { 7. ¿Cómo dirían que se manifiesta el "vacío existencial" en la vida } \\
\text { de los jóvenes? }\end{array}$ & $x$ & $X$ & $X$ & $X$ \\
\hline $\begin{array}{l}\text { 8. ¿Qué situaciones llevan a pensar a los jóvenes universitarios } \\
\text { que su vida carece de sentido? }\end{array}$ & $x$ & $x$ & $x$ & $x$ \\
\hline $\begin{array}{l}\text { 9. ¿Cuáles diría que son los tipos de sentido que tienen los } \\
\text { jóvenes universitarios? }\end{array}$ & $x$ & $x$ & & \\
\hline
\end{tabular}

sujetos. A continuación del registro de intervenciones se presenta la "Sintesis de respuestas", en donde se condensan los factores de sentido presentes en la respuesta a cada pregunta, sobre los que finalmente se realiza la asignación de cada sujeto y de cada pregunta a un tipo de sentido según los criterios de sentido. En el primer criterio DO, Despliegue de radicales RAS y Orientación en líneas existenciales LOS-LOCS, se señalan los radicales antropológicos y las líneas de orientación que el sujeto reporta en sus respuestas. Si presenta al menos uno de los RAS de nivel espiritual, estos radicales se consideran como "desplegados". En cuanto a la orientación existencial, se consignan las líneas al sentido y al contrasentido que el sujeto manifiesta en su respuesta y se restan (LOS-LOCS) para obtener el valor positivo o negativo de la orientación existencial. De esta manera, el sujeto se ubica en uno de los cuadrantes de sentido teniendo en cuenta el signo positivo o negativo en los valores RAS y LOS-LOCS. Si los radicales antropológicos RAS son desplegados (+) y las líneas existenciales LOS positivas (+), corresponde al cuadrante ++ ; si los radicales RAS son inhibidos (-) y los ámbitos LOS positivos (+), corresponde al cuadrante - +; si los RAS son desplegados (+) y los ámbitos LOS negativos (-), se ubica el sujeto en el cuadrante + -; y si los RAS están inhibidos (-) y también las líneas LOS son negativas (-), el sujeto se asigna al cuadrante - - .

El segundo criterio, actitud ante el valor (AV), se define por referencia al primero: si la respuesta del sujeto está en el cuadrante RAS+ LOS+, significa una actitud "objetiva" y en los restantes cuadrantes se considera "subjetiva". El tercer criterio, grado de interés vital (GIV), observa la proporcionalidad entre RAS y LOS (o RAS-LOCS). Si la diferencia es mayor o igual a 2 entre estas variables, o si hay un solo RAS o un solo LOS-LOCS, se considera grado de interés débil. En cambio, se requiere la presencia de 2 LOS $y$ al menos un RAS espiritual para que sea un grado de interés fuerte. Se reconoce también un grado de interés fuerte cuando dicha proporcionalidad, entre el número de RAS y el de LOS, es directa, es decir, 2/2, $3 / 3,4 / 4$, o en todo caso, la diferencia entre uno y otro número en sendas variables es menor o igual a 2; ya sea RAS + y LOS+ o RAS- y LOCS- o RAS + y LOCS- RAS- 
LOS-. En cuanto al cuarto criterio, estabilidad vital (EV), si el sujeto presenta 3 o más LOS o LOCS, se considera "estable"; de lo contrario, será una respuesta vital "inestable".

Una vez determinado el cuadrante por el criterio $\mathrm{DO}$, se establece el tipo de sentido conjugándolo con el criterio de estabilidad vital (EV). El criterio del grado de interés vital (GIV) parece no tener influencia en la determinación del tipo de sentido, pero sugiere una disposición a la migración de tipo de sentido dentro del cuadrante estable-inestable.

Por su parte, el cuestionario permite profundizar de forma individual en la actitud personal de cada sujeto frente al sentido de vida. Cada sujeto cuenta con el tiempo y con la intimidad necesaria para la reflexión que requiere confrontarse a sí mismo (Marías, 1971). Como se puede observar, en general, en cada pregunta del cuestionario se tienen en cuenta dos criterios: la proporción de factores propios de cada pregunta que manifiesta el sujeto (LOS, RAS, satisfacción, frustración, grado de responsabilidad, participación activa, actitudes ante el futuro, cambio, situaciones límite y los tipos de sentido), y el grado de importancia o desarrollo que le otorga a cada uno. La definición de variables de cara a la asignación operativa del tipo de sentido de vida se realiza según el modelo de sentido, como se expresa en la figura 1.

Por otra parte, en la estructura de prueba del cuestionario (cuadro 3) se presentan los distintos pesos que cada criterio tiene en cada pregunta y las observaciones pertinentes para el análisis del investigador.

Cuadro 3. Estructura de prueba del cuestionario “Tipos de sentido de vida en los jóvenes universitarios" (Cadavid y Díaz, 2014)

\begin{tabular}{|c|c|c|c|c|}
\hline \multirow{2}{*}{ Preguntas del cuestionario } & Crt1 & Crt2 & $\mathrm{Crt3}_{3}$ & Crt4 \\
\hline & DO & AV & EV & GIV \\
\hline $\begin{array}{l}\text { 1. Qué grado de importancia tienen en su vida los siguientes aspectos. Califi- } \\
\text { que de } 1 \text { a } 5\end{array}$ & & $x$ & $x$ & \\
\hline $\begin{array}{l}\text { 2. Valore hasta qué nivel ha desarrollado en su vida las siguientes característi- } \\
\text { cas: Nulo-Muy poco-Poco-Suficiente-Mucho-Totalmente }\end{array}$ & & $x$ & & \\
\hline $\begin{array}{l}\text { 3. Señale el grado de importancia que tienen en su vida las siguientes satisfac- } \\
\text { ciones: Ninguno-Muy Bajo-Bajo-Medio-Alto-Muy alto }\end{array}$ & $x$ & $x$ & $x$ & $x$ \\
\hline $\begin{array}{l}\text { 4. ¿Qué aspectos considera que caracterizan una vida frustrada? Señale qué } \\
\text { grado de frustración causarían en su vida cada una de las siguientes situacio- } \\
\text { nes: Ninguno-Muy Bajo-Bajo-Medio-Alto-Muy alto }\end{array}$ & $x$ & $x$ & $x$ & $x$ \\
\hline $\begin{array}{l}\text { 5. ¿Qué grado de responsabilidad cree usted que ejerce en sus decisiones? } \\
\text { Ninguna-Baja-Media-Alta-Muy alta }\end{array}$ & & $x$ & & \\
\hline $\begin{array}{l}\text { 6. ¿Cuál es su grado de participación en los siguientes ámbitos? Ninguno- } \\
\text { Muy Bajo-Bajo-Medio-Alto-Muy alto }\end{array}$ & $x$ & $x$ & $x$ & $x$ \\
\hline $\begin{array}{l}\text { 7. ¿Cuál es su actitud frente al futuro? Señale el grado en que los conceptos } \\
\text { concuerden con su posición: Ninguno-Muy Bajo-Bajo-Medio-Alto-Muy alto }\end{array}$ & $x$ & $x$ & $x$ & $x$ \\
\hline $\begin{array}{l}\text { 8. Si pudiera volver a nacer, ¿qué cambiaría en su vida. Señale el grado de cambio } \\
\text { que quisiera conseguir?: Ninguno-Muy Bajo-Bajo-Medio-Alto-Muy alto }\end{array}$ & $x$ & $x$ & $x$ & $x$ \\
\hline $\begin{array}{l}\text { 9. Qué actitud toma frente a las siguientes situaciones. Señale la que corres- } \\
\text { ponda: Enfrenta-Evade }\end{array}$ & $x$ & $x$ & & \\
\hline $\begin{array}{l}\text { 10. Valore hasta qué punto se identifica con los siguientes tipos de sentido } \\
\text { de vida (se presentan los nueve tipos de sentido de vida con una nominación } \\
\text { comprensible a los universitarios y una breve definición de cada uno): Nula- } \\
\text { Baja-Media-Alta-Muy Alta }\end{array}$ & $x$ & $x$ & $x$ & $x$ \\
\hline
\end{tabular}


El cuestionario se compone de diez preguntas que indagan sobre los ámbitos existenciales, los radicales antropológicos de sentido y los tipos de sentido de vida que manifiestan los jóvenes universitarios (Acevedo, 1998). En general, las preguntas utilizan una gradación de respuesta según la escala de Likert, excepto la pregunta número 9 que por indagar sobre la actitud de evasión o enfrentamiento ante cuatro situaciones vitales más o menos críticas (Jaspers, 2000, p. 22), se comporta en las posibles combinaciones de respuesta no como una variable continua sino discreta.

En el instrumento se cuestiona en primera instancia el grado de importancia o presencia que tienen en la vida del joven universitario los ámbitos existenciales de orientación al sentido (Nicol, 1996), LOS (preguntas 1 y 6 ), y el nivel de desarrollo de los RAS (pregunta 2). Las preguntas 3 y 4 indagan las satisfacciones y frustraciones a partir del despliegue o inhibición de estos radicales correspondientes a tales dinamismos. En la pregunta 5 se contrasta en general el reporte del joven universitario respecto a sus radicales de sentido y especialmente se confronta su autodeterminación. Una exploración particular se realiza con la pregunta 7 sobre el subconjunto de valores de actitud específicos del futuro, tanto positivos como negativos (antivalores) (Restrepo, 2007, p. 454). Este conjunto de valores aporta un núcleo importante de referencia en la configuración existencial del sujeto en líneas de orientación al sentido y al contrasentido (LOS y LOCS). La pregunta 8 constituye una presentación vital para el joven que conjuga radicales y líneas existenciales de sentido para arrojar más claridad sobre los factores de sentido mejor reconocidos por el joven universitario. Del lado del investigador, esta pregunta es de alta significatividad, en tanto lo es el cuestionamiento al joven sobre aquellos ingredientes personales y existenciales que quisiera modificar si volviera a nacer La última pregunta recoge los tipos de sentido con que mejor se identifican los estudiantes según el modelo de sentido de vida propuesto en la investi- gación, si bien se han buscado unos nombres que siendo acordes con la descripción que se presenta en el instrumento puedan tener impacto y significado para los jóvenes.

Hay que anotar que siendo las puntuaciones de las preguntas en general directas respecto al grado de importancia, presencia o desarrollo de los factores de sentido, hay una pregunta que claramente es de puntuación inversa: la 8. Esta explora los cambios que quisiera realizar la persona en el orden vital e íntimo y, por ello, a mayor cambio propuesto menor sentido vital en el estado de cosas actual, e inversamente, a menos cambio "solicitado" mayor presencia de sentido. Otra pregunta con puntuación inversa es la número 7 . En ella, la mitad de las actitudes ante el futuro se plantean en negativo, en correspondencia con las actitudes positivas, lo cual permite una ponderación más completa y exhaustiva de las actitudes ante el futuro. Respecto a la pregunta 4, sobre los tipos de frustración correspondientes a la inhibición de los radicales RAS, pareciera que debiera ofrecer puntuación inversa, pero en realidad no es así ya que, aunque se pregunta por los grados de frustración que generarían distintas situaciones personales, lo que verdaderamente se refleja en las respuestas es la importancia que se da a las condiciones antropológicas de inhibición de los radicales de sentido (Sánchez-Migallón, 2006, pp. 210-211). De este modo, las relaciones implícitas en las respuestas a esta pregunta sobre las distintas frustraciones antropológicas se expresan como una proporcionalidad directa: a mayor grado de frustración reconocido en las distintas situaciones, mayor importancia se confiere personalmente a la inhibición de los radicales antropológicos de sentido en cuestión; por tanto, se observa que, efectivamente, la puntuación que se genera en esta pregunta sí es directa.

\section{Presentación de la experiencia y análisis de resultados}

En los cuadros 4 y 5 se especifica la participación de los sujetos según la aplicación de cada instrumento en la investigación. 


\section{Cuadro 4. Población de las entrevistas focales}

\begin{tabular}{|c|c|c|c|c|c|c|}
\hline Grupos & Universidad & Facultad & N & Mujeres & Hombres & Edad \\
\hline 2 & Javeriana & Ingeniería & 18 & 9 & 9 & $18-22$ \\
\hline 1 & La Sabana & $\begin{array}{c}\text { Comunicación } \\
\text { Administración } \\
\text { Ing. Industrial } \\
\text { Psicología }\end{array}$ & 8 & 5 & 3 & $19-22$ \\
\hline 1 & Minuto de Dios & $\begin{array}{c}\text { Ingeniería } \\
\text { Trabajo social } \\
\text { Administración } \\
\text { Comunicación social }\end{array}$ & 14 & 11 & 3 & $18-25$ \\
\hline 2 & La Salle & $\begin{array}{c}\text { Trabajo social } \\
\text { Optometría } \\
\text { Lenguas Modernas }\end{array}$ & 7 & 6 & 1 & $18-25$ \\
\hline 2 & Católica & $\begin{array}{c}\text { Ingeniería } \\
\text { Economía } \\
\text { Psicología }\end{array}$ & 15 & 11 & 4 & $18-24$ \\
\hline 1 & Distrital & Ingeniería & 19 & 2 & 17 & $18-22$ \\
\hline 11 & Pedagógica Nacional & $\begin{array}{c}\text { Educación } \\
\text { Humanidades }\end{array}$ & 5 & 5 & 0 & $18-27$ \\
\hline
\end{tabular}

\section{Cuadro 5. Población de los cuestionarios}

\begin{tabular}{|c|l|c|}
\hline No. & \multicolumn{1}{|c|}{ Universidad } & No. de sujetos \\
\hline 1 & Javeriana & 99 \\
\hline 2 & La Sabana & 56 \\
\hline 3 & Santo Tomás & 129 \\
\hline 4 & Minuto de Dios & 72 \\
\hline 5 & La Salle & 99 \\
\hline 6 & Católica & 92 \\
\hline 7 & Distrital & 72 \\
\hline 8 & Pedagógica Nacional & 15 \\
\hline & Total & $\mathbf{6 3 4}$ \\
\hline
\end{tabular}

\section{Resultados de las entrevistas en grupos focales}

El encuentro personal con los jóvenes estuvo enmarcado por un ambiente positivo de expectativa acerca del tema por discutir. Al enunciar la primera pregunta, se dispusieron a realizar el trabajo de pensar en el sentido. Al finalizar expresaron que el tema les había parecido sumamente importante y provechoso para sus vidas y que necesitaban más espacios de este tipo para tratar estos temas fundamentales de la existencia humana (Frondizi, 1977).

Se codifica a cada sujeto siguiendo el formato de sintesis de las respuestas de cada pregunta; luego, se procede a realizar el análisis cualitativo de cada grupo focal. En esa primera recolección de datos 
dentro de cada entrevista se relacionó cada respuesta con los cuatro criterios establecidos para el análisis respectivo (despliegue-orientación, actitud ante el valor, estabilidad vital y grado de interés vital). Así se desarrolla el análisis según los criterios de sentido de vida, que permiten un mejor seguimiento metodológico de los factores de sentido que han sido la estructura conceptual por excelencia de esta investigación (Restrepo, 2007, pp. 452-457).

Los factores comunes a todos los grupos de jóvenes de la universidades participantes se manifiestan en que piensan que el sentido de vida está relacionado con alcanzar las metas y los objetivos propuestos, con estar rodeados del apoyo, con la comprensión y el afecto de la familia, con sentirse amados y aceptados por sus pares y también, de modo relevante, con saberse cuidados, queridos y respetados por los docentes. Expresan además lo importante que es el ámbito universitario como fuente de riqueza y de sentido. El sentido de vida lo relacionan los jóvenes con el buen desempeño académico, con exigirse y defender la libre elección de su carrera, sin presiones, y, sobre todo, al hallazgo de la vocación. También hay un sentido y una relación estrecha entre este desempeño académico y el sueño de alcanzar un desarrollo profesional importante en el futuro; asimismo, esta ubicación y competencia laboral la relacionan además con la prestancia y el reconocimiento social que tiene la Universidad en la que estudian. Por otra parte, el espíritu de superación es reconocido por los jóvenes como un factor importante de sentido, en tanto constituye la actitud necesaria para avanzar y sortear las dificultades de la vida.

En contraste con las semejanzas halladas, se presentan algunas caracterizaciones específicas respecto a la configuración del sentido en los jóvenes según cada una de las universidades participantes en la investigación. Por una parte, hay jóvenes que saben bien que dependen de sus familias, pero para ellos estas significan un entorno de protección y seguridad en el presente que les ayuda a su desarrollo como estudiantes universitarios, aunque sienten cierto temor ante el proceso de independencia por venir; unos se saben protegidos por el ámbito familiar, mientras otros se sienten desprotegidos e inseguros durante el proceso universitario, antes de salir al escenario laboral, por las carencias o desajustes de este ámbito. Aparece, además, el temor de algunos jóvenes ante la posibilidad de no lograr ubicarse laboralmente, mientras que otros tienen más seguridad y confianza en su posición para la inserción laboral. Para unos jóvenes, el sentido de su formación profesional está ligado a la proyección social en cuanto al aporte al desarrollo, en cambio otros jóvenes relacionan su actividad profesional de un modo más específico, con su sobrevivencia económica.

En conclusión, predomina el tipo de sentido objetivo inestable, seguido por el sentido objetivo estable, salvo en el caso de una universidad, en donde surge en segundo lugar el contrasentido inestable. En tercer lugar predominan el sentido subjetivo existencial inestable y el contrasentido inestable. Puede decirse que cuando los jóvenes universitarios hablan del sentido en grupo realizan una reflexión positiva con relación a él; esto probablemente debido a que el espacio caracterizado por el ambiente de intimidad y de pregunta les facilita pensar, y la dinámica de intercambio los motiva a manifestar su posición más como colectivo que como singularidad. Además, aunque tanto las preguntas como el tiempo de la entrevista permiten asegurar cierto acercamiento a los factores antropológicos y existenciales del sentido de los jóvenes, también limitan en cierta medida su expresión íntima personal sobre el sentido en sus vidas. Pero ello no impide que los jóvenes desvelen sus dificultades de forma sincera y abierta, unos valorando la experiencia del dolor, otros solicitando alternativas de atención y de ayuda. También se presentan los jóvenes que guardan silencio en algunas preguntas pero en los que se observa la inquietud interior del ponerse a pensar. Sin embargo, el hecho de que predominen los sentidos objetivo inestable seguido del inestable, en este caso del que puede migrar hacia los subjetivos, no excluye la presencia importante del sentido subjetivo y del contrasentido. 
Es relevante anotar cómo la inclusión del joven en la universidad se constituye en un factor de protección que asegura en gran medida su desarrollo personal. Esto especialmente, según ellos lo refieren, cuando tienen la fortuna de encontrarse con docentes con talante de formadores que acogen y promueven el crecimiento humano de sus estudiantes.

Por otro lado, el compañerismo, el apoyo y el afecto de los verdaderos amigos son valores inconmensurables para ellos; asimismo, el afán por surgir profesionalmente va ligado al temor por no poder realizar este logro. Para los jóvenes, la presencia y la calidad de la dinámica familiar constituyen de manera definitiva la base desde la cual se impulsan con mayor o menor facilidad hacia el futuro, requieren de su respaldo, su orientación y la alegría de ser valorados sin restricción. Igualmente, para los jóvenes sigue siendo un anhelo crear una familia en un futuro, piensan en encontrar el amor verdadero, rescatar el matrimonio y tener hijos. Se ven a sí mismos trabajando por el bienestar de sus familias y de la sociedad. Con relación a la actitud religiosa, los jóvenes reconocen el valor de la vida buena, marcada por valores objetivos, y conservan el referente de Dios en sus vidas. Sin embargo, no parecen dispuestos a comprometerse con su religión formalmente.

\section{Resultados del cuestionario}

Con el fin de organizar los datos que surgen de la aplicación de los cuestionarios, se realiza una captura de datos en Excel por cada sujeto y por cada pregunta para ser procesada en el paquete estadístico SPSS.

Se definen los rangos de asignación de variables para cada pregunta y la correspondencia de estos con uno de los nueve tipos de sentido de vida según el marco antropológico precedente a la investigación de campo (Cadavid y Díaz, 2104). De este modo, las respuestas de cada persona se pueden asignar a un determinado tipo de sentido de vida según el número de LOS, RAS, satisfacciones, frustraciones y demás componentes del cuestionario, combinado con los factores de sentido de nivel superior, es decir, el número de LOS de actitud y de RAS espirituales, y el grado de importancia que cada participante les confiere a aquellos.

La pregunta 1 recoge las líneas de orientación al sentido (LOS) que reconoce cada participante para situarse en algún tipo de sentido según el número de LOS que reporta y el grado de importancia que le asigna a cada uno en la escala del cuestionario, y la presencia o no de LOS superiores o de actitud que corresponden al nivel alto más propio del carácter espiritual de la persona (Cadavid y Díaz, 2014). Para el tipo de sentido objetivo estable, corresponde la presencia de 15016 líneas de orientación al sentido (LOS), calificadas por cada persona con grado de importancia igual o superior a 4 y de los cuales por lo menos 5 LOS son de actitud. En el caso del sentido objetivo inestable, la persona reconoce menos LOS manifiestos, entre 13 y 16, con puntuación por encima o igual a 4 y expresa 4 O 5 LOS de actitud. Por otra parte, el sentido subjetivo antropológico inestable se asigna cuando el universitario presenta 11 y 12 LOS en grado 4 o más, con 3, 4 O 5 LOS de actitud; o también cuando el estudiante tiene 13 y 14 LOS en grado 4 o superior, y de ellos solo 3 son de actitud. Así se continúan estableciendo los rangos exigidos para la asignación de cada tipo de sentido. Proceso análogo se desarrolla con las otras preguntas que corresponden a LOS $(6,7$ y 8$)$ y también en las que se contempla el despliegue de radicales antropológicos de sentido (RAS) (en las preguntas 2, 3, 4 y 9). La pregunta 5 funciona más como variable discreta frente a esta asignación del tipo de sentido.

Por otro lado, la pregunta 10 organiza su asignación explícitamente con los tipos de sentido propuestos desde el grado de identificación que realiza cada persona respecto de cada uno. Para la pregunta número 9 se consideran las puntuaciones ponderadas en cada respuesta según la importancia o el peso de cada situación (cuadro 6). Estas condiciones se constituyen en una exigencia sine qua non para la asignación de ese sujeto en un tipo de sentido en particular. 
ISSN 0123-1294 | Educ.Educ. Vol. 18. No. 3 | Septiembre-Diciembre de 2015 | pp. 371-390.

Universidad de La Sabana | Facultad de Educación

Cuadro 6. Asignación de rangos de variables de la pregunta 9- situaciones límite

\begin{tabular}{|l|c|c|}
\hline \multicolumn{1}{|c|}{ Situaciones } & Enfrenta & Evade \\
\hline Ante lo que le ocurre como enfermedades, accidentes, pérdidas o muerte & +4 & -4 \\
\hline Ante las consecuencias de sus acciones y palabras & +1 & -1 \\
\hline Ante las contrariedades y situaciones imprevistas & +2 & -2 \\
\hline Ante las actitudes negativas de los demás & +3 & -3 \\
\hline
\end{tabular}

Los resultados se presentan en tablas y figuras en donde se aplicaron pruebas estadísticas: análisis de varianza (ANOVA), chi-cuadrado para proporciones y coeficientes de correlación. Se consideró significativo $p<0,05$.

La mayor proporción de estudiantes participantes, el $57,9 \%$, fue de sexo femenino; y el grupo etario más frecuente es de 20 a 24 años con el 59,4\%.

A continuación se enuncian los tres grupos de resultados que se consideran más relevantes. De una parte, la clasificación de los tipos de sentido relevantes (objetivo, contrasentido y mayoritarios) de los sujetos según cada una de las preguntas del cuestionario (tabla 2). En segundo lugar, la evaluación global de los tipos de sentido resultantes de todas las respuestas con distribución porcentual de frecuencias según sexo (tabla 3). En tercer lugar, los tipos de sentido de vida totales según los cruces de las preguntas de RAS y de LOS (tabla 4).

En el primer grupo de resultados (tabla 2), se observan las distribuciones de frecuencia de sentido por pregunta según sexo y moda de respuesta. Puede analizarse la posición noógena de sentido (Frankl, 1995) de los jóvenes con predominancia en cada una de las preguntas del cuestionario. Las preguntas más sensibles a las frecuencias del sentido de vida objetivo estable e inestable son: responsabilidad personal ( $82 \%)$, posibles cambios en la vida ( $41 \%)$, satisfacciones personales (37\%), actitudes ante situaciones limite (33\%), frustraciones personales (27\%) y despliegue existencial (21\%). Por su lado, las preguntas que mejor discriminan las frecuencias mayores de contrasentido fueron: participación en ambientes (30\%), disposición personal al sentido (19\%) y frustraciones personales (18\%).

El segundo grupo de resultados consiste en la evaluación global de los tipos de sentido incluyendo las nueve preguntas. Se excluye la pregunta 10 porque su información se trató de manera independiente. Hombres y mujeres se ubican de manera contundente (44,5\%) en el tipo de sentido antropológico estable, estando los hombres más afincados en este sentido (53,5\%) que las mujeres (38,0\%). La

Tabla 1. Distribución porcentual de estudiantes

\begin{tabular}{|l|c|c|}
\hline \multicolumn{1}{|c|}{ Edad agrupada } & $\mathbf{n}$ & \% \\
\hline $17-19$ & 227 & 36,3 \\
\hline $20-24$ & 372 & 59,4 \\
\hline$>=25$ & 27 & 4,3 \\
\hline Total & 626 & 100,0 \\
\hline Sexo femenino & 358 & 57,9 \\
\hline Sexo masculino & 260 & 42,1 \\
\hline Total & 618 & 100,0 \\
\hline
\end{tabular}


Tabla 2. Tipos de sentido de vida por pregunta. Distribución porcentual de frecuencias según sexo

\begin{tabular}{|c|c|c|c|c|c|c|c|}
\hline & \multicolumn{2}{|c|}{ Femenino } & \multicolumn{2}{|c|}{ Masculino } & \multicolumn{2}{|c|}{ Total } & \multirow{2}{*}{$\mathrm{p}$} \\
\hline & $\mathbf{n}$ & $\%$ & $\mathbf{n}$ & $\%$ & $\mathbf{n}$ & $\%$ & \\
\hline \multicolumn{7}{|l|}{ P1. Despliegue existencial } & 0,03 \\
\hline Antropológico estable & 111 & 31,0 & 113 & 43,5 & 224 & 36,2 & \\
\hline Objetivo inestable & 90 & 25,1 & 40 & 15,4 & 130 & 21,0 & \\
\hline \multicolumn{8}{|l|}{ P2. Disposición personal al sentido } \\
\hline Contrasentido límite de vacuidad & 12 & 3,4 & 8 & 3,1 & 20 & 3,4 & \\
\hline Contrasentido estable & 19 & 5,4 & 14 & 5,4 & 33 & 5,6 & \\
\hline Contrasentido inestable & 36 & 10,2 & 28 & 10,8 & 64 & 10,8 & \\
\hline Existencial estable & 84 & 23,7 & 72 & 27,7 & 156 & 26,3 & \\
\hline \multicolumn{8}{|l|}{ P3. Satisfacciones personales } \\
\hline Objetivo inestable & 134 & 37,4 & 97 & 37,5 & 231 & 37,7 & \\
\hline \multicolumn{8}{|l|}{ P4. Frustraciones personales } \\
\hline Contrasentido límite de vacuidad & 30 & 8,4 & 25 & 9,6 & 55 & 9,8 & \\
\hline Contrasentido estable & 14 & 3,9 & 8 & 3,1 & 22 & 3,9 & \\
\hline Contrasentido inestable & 13 & 3,6 & 12 & 4,6 & 25 & 4,4 & \\
\hline Objetivo inestable & 94 & 26,3 & 58 & 22,3 & 152 & 27,0 & \\
\hline \multicolumn{8}{|l|}{ P5. Responsabilidad personal } \\
\hline Objetivo inestable & 191 & 55,5 & 136 & 53,8 & 327 & 54,8 & \\
\hline Objetivo estable & 99 & 28,8 & 68 & 26,9 & 167 & 28,0 & \\
\hline \multicolumn{8}{|l|}{ P6. Participación en ambientes } \\
\hline Contrasentido límite de vacuidad & 14 & 3,9 & 13 & 5,0 & 27 & 4,6 & \\
\hline Contrasentido estable & 37 & 10,3 & 27 & 10,4 & 64 & 10,8 & \\
\hline Contrasentido inestable & 49 & 13,7 & 39 & 15,0 & 88 & 14,9 & \\
\hline Existencial inestable & 72 & 20,1 & 54 & 20,8 & 126 & 21,3 & \\
\hline \multicolumn{8}{|l|}{ P7. Actitud ante el futuro } \\
\hline Antropológico estable & 166 & 46,4 & 127 & 48,8 & 293 & 47,4 & \\
\hline \multicolumn{8}{|l|}{ P8. Posibles cambios en la vida } \\
\hline Objetivo estable & 129 & 36,0 & 125 & 48,1 & 254 & 41,1 & \\
\hline \multicolumn{8}{|c|}{ P9. Actitud ante las situaciones limite } \\
\hline Existencial inestable & 151 & 42,4 & 121 & 46,5 & 272 & 44,2 & \\
\hline Objetivo estable & 119 & 33,4 & 85 & 32,7 & 204 & 33,1 & \\
\hline Total & 356 & 100 & 260 & 100 & 616 & 100 & \\
\hline
\end{tabular}

segunda ubicación predominante a cierta distancia es el sentido antropológico inestable con el $26,1 \%$ del total, correspondiendo a los hombres el 20,8\% y a las mujeres el 29,9\%. En tercer lugar, aparece el sentido existencial inestable con un porcentaje total de $23,6 \%$, correspondiendo el $21,2 \%$ a los hombres y $25,4 \%$ a las mujeres. Esto significa que más del $90 \%$ de los jóvenes universitarios están ubicados en alguno de los tipos sentidos de vida subjetivos. Lo anterior quiere decir que tanto hombres como mujeres 
ISSN 0123-1294 | Educ.Educ. Vol. 18. No. 3 | Septiembre-Diciembre de 2015 | pp. 371-390.

Universidad de La Sabana | Facultad de Educación

presentan radicales antropológicos inhibidos en su mayoría, aunque con cierto despliegue en algunos ámbitos existenciales positivos (LOS). En la tabla 3, de evaluación de los tipos de sentido de vida por sexo, se encuentra una diferencia estadísticamente significativa $(p=0,001)$.

El tercer grupo de resultados se refiere a la evaluación global de las respuestas correspondientes a RAS y LOS. En la tabla 4 se observa el cruce de respuestas a las preguntas de LOS y las preguntas de RAS. Los estudiantes, en cuanto a las preguntas de LOS aparecen mayoritariamente en el antropológico estable y existencial inestable (42,9 y 31,6\%) y en cuanto a RAS se sitúan más en el sentido existencial inestable y estable (35 y 33\%). Se observa la consonancia de los tipos de sentido predominantes en los jóvenes como en el grupo de resultados anterior. Esto puede explicarse porque los jóvenes tienen alguna conciencia de su dignidad personal aunque no consiguen desplegarse en una red rica de ámbitos existenciales. Además, se encontró una correlación entre los tipos de sentido de las preguntas de RAS y las preguntas de LOS de $r=0,43$, lo cual significa que en general hay correlación positiva en la asignación de tipos de sentido de las preguntas de LOS con la asignación de las preguntas de RAS.

\section{Síntesis final y recomendaciones}

Los hombres, en cuanto al sentido de vida, están más arraigados en el plano del sentido construido o inventado, alejado del esfuerzo de descubrimiento de la misión fundamental, más cerca de la trivialidad y de la búsqueda del éxito material. Es notorio el repliegue de sus posibilidades interiores, de sus valores intrínsecos y de su potencialidad espiritual. Los jóvenes están más centrados en lo pragmático, siendo ellos mismos producto de la cultura del resultado que de la calidad del bien vivir cotidiano. Llama la atención el alejamiento de su propia identidad como persona y son más partícipes de los modelos y las exigencias trazados por el entorno. Sin embargo, la investigación encuentra también jóvenes que se disponen a ser consistentes con su propia naturaleza humana libre y responsable y se ven a sí mismos convocados a realizar una misión existencial, aunque esta no signifique un triunfo "socialmente correcto". Se podría inferir una gran permeabilidad en el sentido de vida del joven de la

Tabla 3. Evaluación global de los tipos de sentido de vida: distribución porcentual de frecuencias según sexo

\begin{tabular}{|c|c|c|c|c|c|c|c|}
\hline & & $\mathrm{Se}$ & & & & & $n$ \\
\hline & & & & lino & & & $P$ \\
\hline & $\mathrm{n}$ & $\%$ & $\mathbf{n}$ & $\%$ & $\mathbf{n}$ & $\%$ & \\
\hline Contrasentido límite de vacuidad & $\mathrm{O}$ & 0,0 & $\mathrm{o}$ & 0,0 & o & 0,0 & 0,001 \\
\hline Contrasentido estable & $\mathrm{O}$ & 0,0 & $\mathrm{o}$ & 0,0 & $\mathrm{o}$ & 0,0 & \\
\hline Contrasentido inestable & 4 & 1,1 & 0 & 0,0 & 4 & 0,6 & \\
\hline Existencial estable & 12 & 3,4 & 11 & 4,2 & 23 & 3,7 & \\
\hline Existencial inestable & 91 & 25,4 & 55 & 21,2 & 146 & 23,6 & \\
\hline Antropológico estable & 136 & 38,0 & 139 & 53,5 & 275 & 44,5 & \\
\hline Antropológico inestable & 107 & 29,9 & 54 & 20,8 & 161 & 26,1 & \\
\hline Objetivo inestable & 8 & 2,2 & 1 & 0,4 & 9 & 1,5 & \\
\hline Objetivo estable & o & 0,0 & $\mathrm{O}$ & 0,0 & $\mathrm{O}$ & 0,0 & \\
\hline Total & 358 & 100,0 & 260 & 100,0 & 618 & 100,0 & \\
\hline
\end{tabular}


Tabla 4. Distribución cruzada de frecuencias: tipos de sentidos de vida según preguntas de LOS y de RAS

\begin{tabular}{|c|c|c|c|c|c|c|c|c|c|}
\hline & \multirow[b]{2}{*}{$r=0,43$} & \multicolumn{8}{|c|}{ Tipos de sentido según $\operatorname{LOS}(1,6,7$ y 8$)$} \\
\hline & & 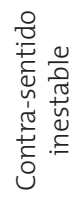 & 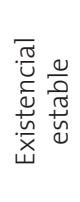 & 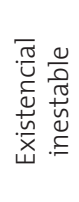 & 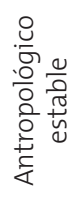 & 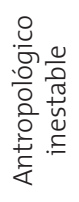 & 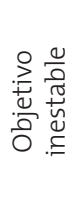 & $\begin{array}{l}\overline{\widetilde{\pi}} \\
\stackrel{0}{\circ} \\
\end{array}$ & ১ \\
\hline \multirow{9}{*}{$\begin{array}{l}\text { Tipos de sentido según RAS } \\
\qquad(2,3,4 \text { y } 9)\end{array}$} & Contrasentido inestable & o & 1 & o & o & $\mathrm{O}$ & o & 1 & 0,2 \\
\hline & Antropológico estable & 4 & 6 & 6 & 3 & 1 & $\mathrm{O}$ & 20 & 3,3 \\
\hline & Antropológico inestable & 1 & 17 & 51 & 17 & 7 & o & 93 & 15,4 \\
\hline & Existencial estable & 2 & 17 & 74 & 81 & 26 & 0 & 200 & 33,2 \\
\hline & Existencial inestable & o & 7 & 49 & 116 & 39 & 1 & 212 & 35,2 \\
\hline & Objetivo inestable & o & 1 & 10 & 41 & 21 & 2 & 75 & 12,5 \\
\hline & Objetivo estable & o & $\mathrm{o}$ & o & o & 1 & o & 1 & 0,2 \\
\hline & Total & 7 & 49 & 190 & 258 & 95 & 3 & 602 & 100 \\
\hline & $\%$ & 1,2 & 8,1 & 31,6 & 42,9 & 15,8 & 0,5 & 100 & \\
\hline
\end{tabular}

cultura del consumo y del amor ligero, del inmediatismo, de un reivindicado individualismo en tensión con su energía vital y su anhelo de progreso y felicidad (Rojas, 1998, pp. 178-180).

Por otra parte, las mujeres, en su mayoría, también están manifestando su sentido de vida en esta línea. Lo cual no sorprende porque es culturalmente apreciable en el entorno de una urbe como Bogotá, altamente influenciada por los medios masivos de comunicación y por el discurso feminista dominante (Taylor, 2006), cómo la mujer, de forma paradójica, tiende a parecerse al hombre más que a reivindicar su distinción femenina en los ámbitos de sentido y en su desenvolvimiento existencial (Marías, 1987, p. 315). Sin embargo, a diferencia de los hombres, en las jóvenes aparece también, aunque no de modo relevante, el sentido de vida basado en valores objetivos, tanto por el arraigo a su propia naturaleza humana como en los ámbitos existenciales que tienen un contenido valioso en términos de descubrimiento de auténtico sentido.

Los jóvenes presentan un riesgo importante de contrasentido debido a la falta de conocimiento de sí mismos y de la debilidad en la capacidad para examinarse y proponerse cambios positivos; así como también, por la incapacidad para realizarlos, debido a la "anorexia" de sentido, a la dificultad en la apertura valiente para encontrar el valor de sí mismos y para responder por ese valor personal en cada decisión de su existencia. Por esta razón, y por la soledad del joven traducida en abandono afectivo y real, por la ausencia de los ámbitos de acogida familiar y social, incluido el universitario, los jóvenes no se encuentran fortalecidos para enfrentar las frustraciones que constituyen una gran posibilidad de diluirse en un vacío de sentido (Lukas, 2004). Parece que al no recibir el alimento del sentido proveniente de los adultos y del entorno social en general, y a través de referentes personales claros y representativos de los valores objetivos (Acevedo y Battafarano, 2003), los jóvenes no se disponen a ofrecer de sí mismos lo que no han recibido, y por tanto están todavía con ansia de tal "nutrición" (Oro, 2003).

Sin embargo, un buen grupo de jóvenes es poseedor de un sentido objetivo de la vida. No lo inventan sino que descubren la verdad en cada realidad de su existencia e intentan responder a ella de la 
mejor manera al elegir las alternativas más adecuadas en cada caso. En esta misión existencial los jóvenes manifiestan mantener una tensión existencial buena que los catapulta siempre a avanzar en su desarrollo como personas y que incluye esfuerzos, y por qué no, hasta sacrificios, entrega de símismos y renuncia a varios placeres inmediatos para conquistar el despliegue más pleno de su potencialidad humana (Guerra, 2002). Así, se aprecia que realizan un modo más humano de existir entretejido con el gozo de la vida y de sus gustos personales: con el placer de ser amigos y miembros de una familia, de ser estudiantes entusiasmados con la excelencia en su profesión y de ser ciudadanos comprometidos con el progreso auténtico de la patria y del mundo global al que pertenecen. Estos jóvenes respiran esperanza y alegría, y saben que hay que sobreponerse para superar las circunstancias adversas que trae la vida. Están reverberantes de sueños, los defienden y saben que la fe, el trabajo arduo y el ser con otros los hacen posibles (Naval, 2001). Es esta una expresión de caminos de sentido para la plenitud de una vida lograda en los jóvenes.
La recomendación de esta investigación consiste en realizar una intervención preventiva en el ámbito educativo universitario que permita fortalecer en los jóvenes los factores antropológicos y existenciales de sentido de vida, impulsar su investigación, así como promover un ambiente académico formativo en lo humano que facilite a los jóvenes un proceso constante de migración y desarrollo hacia el descubrimiento de sentido de vida (López, 2003). Esto debido a que la mayoría, como hemos dicho antes, se ubica en algún tipo de sentido subjetivo, que alerta porque significa la presencia de factores de riesgo de vacío existencial. Por otra parte, es importante anotar que existe una presencia de estudiantes en contrasentido que exige una atención inminente.

Los jóvenes universitarios tienen ante sí el reto de descubrir y reivindicar su identidad y de desplegarse en plenitud de acuerdo con quiénes son y con quiénes quieren llegar a ser (Vial, 2000). La universidad, por su parte, tiene el compromiso de acoger a cada estudiante en su singularidad y acompañarlo de forma benevolente en los procesos de culminar y superar la primera juventud.

\section{Referencias}

Abaggnano, N. (1997). Introducción al existencialismo. Bogotá: Fondo de Cultura Económica.

Acevedo, J. (1998). La búsqueda de sentido y su efecto terapéutico. Buenos Aires: Fundación Argentina de Logoterapia.

Acevedo, J.y Battafarano, M. (2003). Desde Viktor Frankl. Hacia un enfoque transdisciplinario del enfermar humano. Buenos Aires: Fundación Argentina de Logoterapia.

Ander-Egg, E. (2001). Métodos y técnicas de investigación social. I. Acerca del conocimiento y del pensar cientifico. Buenos Aires: Lumen.

Cadavid, M. V.y Díaz, V. M. (2014). Modelo antropológico-existencial del sentido de vida. Pensamiento y Cultura. Revista de Filosofía, 17 (2), pp. 145-180.

Castro, L., Castro, M. A. y Morales, J. (2005). Metodología de las ciencias sociales. Una introducción crítica. Madrid: Tecnos. 
Frankl, V. E. (1984). Psicoterapia y humanismo. ¿Tiene un sentido la vida? México: Fondo de Cultura Económica.

Frankl, V. E. (1995). Psicoterapia al alcance de todos. Barcelona: Herder.

Frankl, V. E. (2001). Psicoterapia y existencialismo. Escritos selectos sobre logoterapia. Barcelona: Herder.

Frankl, V. E. (2003). La idea psicológica de hombre. Madrid: Rialp.

Freire, J. B. (2007). El humanismo de la logoterapia de Viktor Frankl. La aplicación del análisis existencial en la orientación personal. Pamplona: Eunsa.

Frondizi, R. (1977). Introducción a los problemas fundamentales del hombre. México: Fondo de Cultura Económica.

García-Alandete, J., Rosa, E.y Sellés, P. (2013). Estructura factorial y consistencia interna de una versión española del Purpose-In-Life Test. Universitas Psychological, 12 (2), 517-530.

García Pintos, C. et al. (2007). Frankl por definición. Consultor temático de logoterapia y análisis existencial. Buenos Aires: San Pablo.

Guerra, R. (2002). Volver a la persona. El método filosófico de Karol Wojtyla. Madrid: Caparrós.

Heidegger, M. (1998). El ser y el tiempo. México: FCE.

Jaspers, K. (2000). La filosofía: desde el punto de vista de la existencia. México: Fondo de Cultura Económica.

Lara, W. de y Ruschel, D. (2012). Bem-estar psicológico: definição, avaliação e principais correlatos. Estudos de psicologia, 29 (4). Recuperado el 24 de septiembre de 2013 de http://www.scielo.br/scielo.php?script=sci_ arttext\&pid=S0103-166X2012000400013\&lang=pt

López, A. (2003). La cultura y el sentido de la vida. Madrid: Rialp.

Lukas, E. (1994). Para validar la logoterapia. En Frankl, V. E., La voluntad de sentido. Escritos selectos sobre logoterapia. Barcelona: Herder.

Lukas, E. (2003). Logoterapia. La búsqueda de sentido. Barcelona: Paidós.

Lukas, E. (2004). Equilibrio y curación a través de la logoterapia. Barcelona: Paidós.

Marías, J. (1987). La felicidad humana. Madrid: Alianza Editorial.

Marías, J. (1971). Tres versiones de la vida humana. Madrid: Salvat-Alianza.

Martínez Miguélez, M. (2006). Ciencia y arte en la metodología cualitativa. México: Trillas.

Martínez Ortiz, E., Trujillo, A. M., Díaz, J. P.y Jaimes, J. (2011). Desarrollo y estructura de la escala dimensional del sentido de vida. Acta Colombiana de Psicología, 14 (2), 113-119. 
ISSN 0123-1294 | Educ.Educ. Vol. 18. No. 3 | Septiembre-Diciembre de 2015 | pp. 371-390.

Universidad de La Sabana | Facultad de Educación

Martínez, E. R., García-Alandete, J., Sellés Nohales, P., Bernabé, G. y Soucase, B. (2012). Análisis factorial confirmatorio de los principales modelos propuestos para el purpose-in-life test en una muestra de universitarios españoles. Acta Colombiana de Psicología, 15 (1), 67-76.

Naval, C. (2001). Confiar: cuna de la sociabilidad humana. San José: Ediciones Promesa.

Nicol, E. (1996). Psicología de las situaciones vitales. México: FCE.

Oro, O. R. (2003). Persona y personalidad. Distintos enfoques a partir de una visión de conjunto. Buenos Aires: Fundación Argentina de Logoterapia.

Polo, L. (2005). Lo radical y la libertad. Cuadernos de Anuario filosófico, 179. Pamplona: Universidad de Navarra.

Restrepo, A. (2003). Viktor Emil Frankl y la teoría logoterapéutica: reencuentro con la humanidad del hombre. Medellín: Universidad Pontificia Bolivariana.

Restrepo, A. (2007). Triadas franklianas. En García Pintos, C. et al., Frankl por definición. Consultor temático de logoterapia y análisis existencial (pp. 453-457). Buenos Aires: San Pablo.

Rojas, E. (1998). Educación de la voluntad. Madrid:Temas de Hoy.

Sánchez-Migallón, S. (2006). La persona humana y su formación en Max Scheler. Madrid: Eunsa.

Sellés Dauder, J. F. (2007). Antropología para inconformes. Madrid: Rialp.

Spranger, E. (1968). Psicología de la edad juvenil. Madrid: Revista de Occidente.

Taylor, Ch. (2006). Imaginarios sociales modernos. Barcelona: Paidós.

Vial, W. (2000). La antropología de Víktor Frankl. Santiago de Chile: Editorial Universitaria.

Wojtyla, K. (2005). El hombrey su destino. Madrid: Palabra. 